\title{
Characterization Theorem of Generalized Operators
}

\author{
Mahmmoud Salih*, Sulieman Jomah \\ School of Mathematics and Statistics, Northwest Normal University, Lanzhou, China \\ Email: ^mahmmoud.salih@yahoo.com, suliemanjomah@hotmail.com
}

How to cite this paper: Salih, M. and Jomah, S. (2018) Characterization Theorem of Generalized Operators. Journal of Applied Mathematics and Physics, 6, 1434-1442. https://doi.org/10.4236/jamp.2018.67120

Received: May 12, 2018

Accepted: July 10, 2018

Published: July 13, 2018

Copyright $\odot 2018$ by authors and Scientific Research Publishing Inc. This work is licensed under the Creative Commons Attribution International License (CC BY 4.0).

http://creativecommons.org/licenses/by/4.0/

\begin{abstract}
In this paper, by using the $\mathrm{W}$-transform of an operator on white noise functionals, we establish a general characterization theorem for operators on white noise functionals in term of growth condition. We also discuss convergence of operator sequences.
\end{abstract}

\section{Keywords}

White Noise Functionals, W-Transform, S-Transform, Characterization Theorem

\section{Introduction}

The main purpose of the present paper is to obtain the characterization theorem for operators on white noise functionals in term of their W-transform was introduced by authors in [1], and give a criterion for the convergence of operators on white noise functionals in term of their $\mathrm{W}$-transform. In [1] the authors deal with the standard Hida-Kubo-Takenka space, in this paper we deal with the Kondrateiv-Streit space, which is more suitable for our purpose.

On the other hand, the white noise calculus (or analysis) was launched out by Hida [2] in the Gaussian case, with his celebrated lecture notes. The concept of the symbol of an operator is of fundamental importance in the theory of operator on white noise functionals. Obata [3] proved an analytic characterization theorem for symbols of operators on white noise functionals, which is an operator version of the characterization theorem for white noise functionals (see, e.g., [4] [5] [6] [7]). Recently a Characterization theorem of operators of discrete-time normal martingales, was established in [8].

This paper is organized as follows. Section 2, is dedicated to a quick review of white noise functionals. In Section 3, we prove the characterization theorems for 
operators on white noise functionals in term of their W-transform. Finally in Section 4 , convergence of operators is discussed.

\section{Preliminary Result on White Noise}

We start with the real Gelfand triple

$$
E \subset H \subset E^{*} .
$$

The norm of $H$ is denoted by ||$_{0}$ and since compatible the real inner product of $H$ and the canonical bilinear form on $E^{*} \times E$ are denoted by the same symbol $\langle.,$.$\rangle . Suppose \mu$ is the standard Gaussian measure on $E^{*}$ and $L^{2}\left(E^{*}, \mu\right)$ the Hilbert space of $\mathbb{C}$-valued $L^{2}$-function on $E^{*}$. The Winer -Itô-Segal theorem say that $L^{2}\left(E^{*}, \mu\right)$ is unitary isomorphic to Boson Fock space $\Gamma\left(H_{\mathbb{C}}\right)$. The isomorphism is a unique linear extension of the following correspondence between exponential functions and exponential vector:

$$
\phi_{\xi}=\mathrm{e}^{\langle x, \xi\rangle-\langle\xi, \xi\rangle / 2} \leftrightarrow\left(1, \xi, \frac{\xi^{\otimes^{2}}}{2 !}, \cdots, \frac{\xi^{\otimes^{n}}}{n !}, \cdots\right) .
$$

If $\phi \in L^{2}\left(E^{*}, \mu\right)$ and $\left(f_{n}\right)_{n=0}^{\infty} \in \Gamma\left(H_{\mathbb{C}}\right)$ are related through the Wiener-Itô-Segal isomorphism, we write

$$
\phi \sim\left(f_{n}\right)
$$

for simplicity. It is then noted that

$$
\|\phi\|_{0}^{2}=\sum_{n=0}^{\infty} n !\left|f_{n}\right|_{0}^{2},
$$

where $\|\phi\|_{0}$ is the $L^{2}$-norm of $\phi \in L^{2}\left(E^{*}, \mu\right)$.

In order to introduce white noise distribution, we need a particular family of seminorms defining the topology of $E$. By means of the differential operator $A=1+t^{2}-\mathrm{d}^{2} / \mathrm{d} t^{2}$ we introduce a sequence of norms in $H_{\mathbb{C}}$ in such a way that $|\xi|_{p}=\left|A^{p} \xi\right|_{0}$. The number:

$$
0<\rho=\left\|A^{-1}\right\|_{o p}=\frac{1}{2}<1, \delta=\left\|A^{-1}\right\|_{H s}
$$

are frequently used. Suppose $E_{p}$ is the Hilbert space obtained by completing $E$ with respect to the norm $|\cdot|_{p}$. Then it is known that

$$
E \cong \operatorname{proj} \lim _{p \rightarrow \infty} E_{p}, E^{*} \cong \text { ind } \lim _{p \rightarrow \infty} E_{-p} .
$$

The norm is naturally extended to the tensor product $E^{\otimes^{n}}$ and their complexification $E_{\mathbb{C}}^{\otimes^{n}}$. The canonical bilinear form $\langle. .$.$\rangle is also extended to a$ $\mathbb{C}$-bilinear form on $\left(E_{\mathbb{C}}^{\otimes^{n}}\right)^{*} \times\left(E_{\mathbb{C}}\right)^{\otimes^{n}}$.

Let $0 \leq \beta<1$ be a fixed number. For $p \geq 0$, define

$$
\|\phi\|_{p, \beta}^{2}=\sum_{n=0}^{\infty}(n !)^{1+\beta}\left|f_{n}\right|_{p}^{2}, \phi \sim\left(f_{n}\right) .
$$

For each $p \geq 0,(E)_{\beta}=\left\{\phi,\|\phi\|_{p, \beta}<\infty\right\} \quad$ becomes a Hilbert space. We put 


$$
(E)_{\beta}=\operatorname{proj} \lim _{p \rightarrow \infty}\left(E_{p}\right)_{\beta},
$$

which becomes a countable Hilbert nuclear space. Next, we consider the dual spaces. For $0 \leq \beta<1$ and $p \geq 0$, define

$$
\|\phi\|_{-p,-\beta}^{2}=\sum_{n=0}^{\infty}(n !)^{1-\beta}\left|f_{n}\right|_{-p}^{2}, \phi \sim\left(f_{n}\right) .
$$

Then $\|\cdot\|_{-p,-\beta}^{2}$ is a Hilberatian norm on $L^{2}\left(E^{*}, \mu\right)$ and we denote by $\left(E_{-p}\right)_{-\beta}$ the completion. The dual spaces of $(E)_{\beta}$ is given by

$$
(E)_{\beta}^{*} \cong \text { ind } \lim _{p \rightarrow \infty}\left(E_{-p}\right)_{-\beta}=\bigcup_{p \geq 0}\left(E_{-p}\right)_{-\beta} \text {, }
$$

and we come to a complex Gelfand triple:

$$
(E)_{\beta} \subset L^{2}\left(E^{*}, \mu\right) \subset(E)_{\beta}^{*} .
$$

Spaces $(E)_{\beta}$ and $(E)_{\beta}^{*}$ are called spaces of test functions and generalized functions, respectively. The construction of these spaces is due to Kondaratiev and Streit [9]. The canonical bilinear form on $(E)_{\beta}^{*} \times(E)_{\beta}$ will be denoted by $\langle.,$.$\rangle . Then$

$$
\langle\Phi, \phi\rangle=\sum_{n=0}^{\infty} n !\left\langle F_{n}, f_{n}\right\rangle, \Phi \sim\left(F_{n}\right) \in(E)_{\beta}^{*}, \phi \sim\left(f_{n}\right) \in(E)_{\beta} .
$$

For $\xi \in E_{\mathbb{C}}$, define the renormalized exponential function $: \mathrm{e}^{\left\langle, \xi^{\xi}\right\rangle}$ : by

$$
: \mathrm{e}^{\langle, \cdot \xi\rangle}:=\sum_{n=0}^{\infty} \frac{1}{n !}\left\langle: \otimes^{\otimes^{n}}:, \xi^{\otimes^{n}}\right\rangle .
$$

Moreover,

$$
: \mathrm{e}^{\langle,, \xi\rangle}:=\mathrm{e}^{\langle, \xi \xi\rangle-\langle\xi, \xi\rangle / 2} .
$$

It is a fact that $: e^{\langle, ., \xi\rangle}:$ is a test function in $(E)_{\beta}$ for any $\xi \in E_{\mathbb{C}}$.

Definition 2.1. The S-transform of a generalized function $\Phi \in(E)_{\beta}^{*}$ is defined to be the function

$$
S \Phi(\xi)=\left\langle\left\langle\Phi,: \mathrm{e}^{\langle\cdot, \xi\rangle}:\right\rangle\right\rangle, \xi \in E_{\mathbb{C}} .
$$

A fundamental theorem in white noise analysis is the Kondratiev-Streit characterization theorem [9] (see also [10]).

Theorem 2.2. ([10]) The S-transform $F=S \Phi$ of $\Phi \in(E)_{\beta}^{*}$ satisfies the following conditions:

1) For any $\xi$ and $\eta$ in $E_{\mathbb{C}}$, the function $F(z \xi+\eta)$ is an entire function of $z \in \mathbb{C}$.

2) There exists nonnegative constant $K$, $a$ and $p$ such that

$$
|F(\xi)| \leq K \exp \left[a|\xi| \frac{2}{1-\beta}\right], \forall \xi \in E_{\mathbb{C}} .
$$

Conversely, suppose a $\mathbb{C}$-valued function $F$ defined on $E_{\mathbb{C}}$ satisfies the above two conditions. Then there exists a unique $\Phi \in(E)_{\beta}^{*}$ such that $F=S \Phi$ 
and for any $q$ satisfying the condition that

$$
\mathrm{e}^{2}\left(\frac{2 a}{1-\beta}\right)^{1-\beta}\left\|A^{-(q-p)}\right\|_{H s}^{2}<1,
$$

the following inequality holds:

$$
\|\Phi\|_{-q,-\beta} \leq K\left(1-\mathrm{e}^{2}\left(\frac{2 a}{1-\beta}\right)^{1-\beta}\left\|A^{-(q-p)}\right\|_{H s}^{2}\right)^{-\frac{1}{2}}
$$

Theorem 2.3. ([10]) Let $F$ be a function on $E_{\mathbb{C}}$ satisfying the conditions:

1) For any $\xi$ and $\eta$ in $E_{\mathbb{C}}$, the function $F(z \xi+\eta)$ is an entire function of $z \in \mathbb{C}$.

2) There exists positive constant $K$, $a$ and $p$ such that

$$
|F(\xi)| \leq K \exp \left[a|\xi|_{-p}^{\frac{2}{1+\beta}}\right], \forall \xi \in E_{\mathbb{C}} .
$$

Then there exists a unique $\varphi \in\left(E_{q}\right)_{\beta}$ such that $F=S \Phi$ for any $q \in[0, \infty)$ satisfying the condition

$$
\mathrm{e}^{2}\left(\frac{2 a}{1+\beta}\right)^{1+\beta}\left\|A^{-(q-p)}\right\|_{H s}^{2}<1
$$

and

$$
\|\varphi\|_{q, \beta} \leq K\left(1-\mathrm{e}^{2}\left(\frac{2 a}{1+\beta}\right)^{1+\beta}\left\|A^{-(q-p)}\right\|_{H s}^{2}\right)^{-\frac{1}{2}}
$$

\section{White Noise Operator}

Let $L\left((E)_{\beta},(E)_{\beta}^{*}\right)$ (resp. $\left.L\left((E)_{\beta},(E)_{\beta}\right)\right)$ denote the space of all continuous linear operator from $(E)_{\beta}$ into $(E)_{\beta}^{*}$ (resp. $\left.(E)_{\beta}\right)$. In this section, we shall prove a characterization theorem for an operator $\Xi \in L\left((E)_{\beta},(E)_{\beta}^{*}\right)$ and for an operator $\Xi \in L\left((E)_{\beta},(E)_{\beta}\right)$.

The W-transform of an operator $\Xi \in L\left((E)_{\beta},(E)_{\beta}^{*}\right)$ is defined to be an $(E)_{\beta}^{*}$-valued function on $E_{\mathbb{C}}$ defined by

$$
W \Xi(\xi)=\Xi \varphi_{\xi}, \xi \in E_{\mathbb{C}} .
$$

Note that the W-transform is injective and that for any $\phi \in(E)_{\beta}$ and $\xi, \eta \in E_{\mathbb{C}}$, we have $\langle W \Xi(z \xi+\eta), \phi\rangle=S\left(\Xi^{*} \phi\right)(z \xi+\eta), z \in \mathbb{C}$, where $\Xi^{*}$ is the adjoint operator of $\Xi$, i.e., $\Xi^{*}$ is the continuous linear operator from $(E)_{\beta}$ into $(E)_{\beta}^{*}$ such that

$$
\langle\langle\Xi \phi, \psi\rangle\rangle=\left\langle\left\langle\Xi^{*} \psi, \phi\right\rangle\right\rangle, \phi, \psi \in(E)_{\beta} .
$$

It follows from Theorem 2.2 that the function $z \rightarrow\langle\langle W \Xi(z \xi+\eta), \phi\rangle\rangle$ is an entire function on $\mathbb{C}$.

We note that there exist $p \geq 0$ and $K$ such that

$$
\|\Xi \phi\|_{-p,-\beta} \leq K\|\phi\|_{p, \beta}, \phi \in(E)_{\beta} .
$$


Then, we have the following growth condition

$$
\|W \Xi\|_{-p,-\beta} \leq K \exp \left[a|\xi| \frac{2}{1-\beta}\right], \xi \in E_{\mathbb{C}},
$$

where $K=C 2^{\beta}$ and $a=(1-\beta)^{\frac{2 \beta-1}{1-\beta}}$

Theorem 4.1. Let $G$ be an $(E)_{\beta}^{*}$-valued function on $E_{\mathbb{C}}$. Then there exists a continuous operator $\Xi \in L\left((E)_{\beta},(E)_{\beta}^{*}\right)$ such that $G$ is the W-transform of $\Xi$ if and only if $G$ satisfies the following conditions:

1) For each $\xi, \eta \in E_{\mathbb{C}}$, and $\phi \in(E)_{\beta}$ the function $z \rightarrow\langle\langle G(z \xi+\eta), \phi\rangle\rangle$ is an entire function on $\mathbb{C}$.

2) There exist nonnegative constant $K$, $a$ and $p$ such that

$$
\|G(\Xi)\|_{-p,-\beta} \leq K \exp \left[a|\xi| \frac{2}{1-\beta}\right], \forall \xi \in E_{\mathbb{C}} .
$$

Proof. In case of $\beta=0$ the proof is given in [1], the proof for general case $0 \leq \beta<1$ is a simple modification. In fact, the first assertion was shown above. Conversely, suppose $G$ is an $(E)_{\beta}^{*}$-valued function on $E_{\mathbb{C}}$ satisfying (1) and (2), we need only to prove the existence of $\Xi$, fix an arbitrary $\phi \in(E)_{\beta}$. Define a $\mathbb{C}$-valued function $F_{\phi}$ by

$$
F_{\phi}(\xi)=\langle\langle G(\xi), \phi\rangle\rangle, \xi \in E_{\mathbb{C}} .
$$

Then $F_{\phi}$ satisfies (1) and (2) in Theorem 2.2 , clearly for any $\xi, \eta \in E_{\mathbb{C}}$, the function $F_{\phi}(\xi)=\langle\langle G(\xi), \phi\rangle\rangle$ of $z \in \mathbb{C}$ is holomorphic on $\mathbb{C}$ and we have, for $\xi \in E_{\mathbb{C}}$

$$
\left|F_{\phi}\right| \leq\|G(\xi)\|_{-q,-\beta}\|\phi\|_{q, \beta} \leq\left(K\|\phi\|_{q, \beta}\right) \exp \left(a|\xi|_{p}^{\frac{2}{1-\beta}}\right) .
$$

Hence, by Theorem 2.2, there exists a unique $\Phi_{\phi} \in(E)_{\beta}^{*}$ such that

$$
S \Phi_{\phi}(\xi)=F_{\phi}(\xi)=\langle\langle G(\xi), \phi\rangle\rangle, \xi \in E_{\mathbb{C}} .
$$

Moreover, for any $r>p$ with

$$
\begin{gathered}
e^{2}\left(\frac{2 a}{1-\beta}\right)^{1-\beta}\left\|A^{-(r-p)}\right\|_{H s}^{2}<1 \\
\left\|\Phi_{\phi}\right\|_{-r,-\beta} \leq K\|\Phi\|_{q, \beta}\left(1-\mathrm{e}^{2}\left(\frac{2 a}{1-\beta}\right)^{1-\beta}\left\|A^{-(r-p)}\right\|_{H s}^{2}\right)^{-1 / 2} .
\end{gathered}
$$

Hence, the operator $\phi \rightarrow \Phi_{\phi}$ is continuous linear operator from $\left(E_{q}\right)_{\beta}$ into $\left(E_{r}\right)_{\beta}^{*}$, and we obtain $\Xi \in L\left((E)_{\beta},(E)_{\beta}^{*}\right)$ with $\Xi \varphi_{\xi}=G(\xi)$.

Definition 4.2. For $\Xi \in L\left((E)_{\beta},(E)_{\beta}^{*}\right)$ a function on $E_{\mathbb{C}} \times E_{\mathbb{C}}$ is defined by

$$
\hat{\Xi}(\xi, \eta)=\left\langle\left\langle\Xi \phi_{\xi}, \phi_{\eta}\right\rangle\right\rangle, \xi, \eta \in E_{\mathbb{C}}
$$

is called the symbol of $\Xi$.

Corollary 4.3. Suppose that a $\mathbb{C}$-valued function on $E_{\mathbb{C}} \times E_{\mathbb{C}}$ satisfies the following condition: 
1) For each $\xi, \xi^{\prime}, \eta$ and $\eta^{\prime}$ in $E_{\mathbb{C}}$, the function $(z, w) \rightarrow F\left(z \xi+\xi^{\prime}, w \eta+\eta^{\prime}\right)$ is an entire function on $\mathbb{C} \times \mathbb{C}$.

2) There exist $p \geq 0, a>0$ and $K>0$ such that

$$
|F(\xi, \eta)| \leq K \exp a\left(|\xi|_{p}^{\frac{2}{1-\beta}}+|\eta|_{p}^{\frac{2}{1-\beta}}\right), \xi, \eta \in E_{\mathbb{C}} .
$$

Then there exists a unique $\Xi \in L\left((E)_{\beta},(E)_{\beta}^{*}\right)$ such that $F$ is the symbol of $\Xi$. The proof given in $[1,8, \mathrm{p} .91]$ for case of $\beta=0$ is adjust to the general case $0 \leq \beta<1$, see [7].

The W-transform of an operator $\Xi \in L\left((E)_{\beta},(E)_{\beta}\right)$ is defined to be an $(E)_{\beta}$-valued function on $E_{\mathbb{C}}$ defined by

$$
W \Xi(\xi)=\Xi \varphi_{\xi}, \xi \in E_{\mathbb{C}} .
$$

Then for any $\Phi \in(E)_{\beta}^{*}$ and $\xi, \eta \in E_{\mathbb{C}}$, we see that

$$
\langle\langle\Phi, W(z \xi+\eta)\rangle\rangle=S\left(\Xi^{*} \Phi\right)(z \xi+\eta), z \in \mathbb{C},
$$

therefore $z \rightarrow\langle\langle\Phi, W(z \xi+\eta)\rangle\rangle$ is holomorphic on $\mathbb{C}$. Moreover, note that for each $q \geq 0$ there exist $p \geq 0$ and $K>0$ such that

$$
\|\Xi \phi\|_{q, \beta} \leq K\|\phi\|_{p, \beta}, \phi \in(E)_{\beta} .
$$

In particular, for all $\xi \in E_{\mathbb{C}}$

$$
\|W \Xi\|_{q, \beta} \leq K \exp \left[a|\xi|_{p}^{\frac{2}{1+\beta}}\right], \xi \in E_{\mathbb{C}} .
$$

Theorem 4.4. Let $G$ be an $(E)_{\beta}$-valued function on $E_{\mathbb{C}}$, satisfying the following conditions:

1) For each $\xi, \eta \in E_{\mathbb{C}}$, and for any $\phi \in(E)_{\beta}^{*}$ the function $z \rightarrow\langle\langle\phi, G(z \xi+\eta)\rangle\rangle$ is an entire function on $\mathbb{C}$.

2) For any $q \geq 0$, there exist $p \geq 0, a>0$ and $K>0$ such that

$$
\|G(\Xi)\|_{q, \beta} \leq K \exp \left[a|\xi|_{p}^{\frac{2}{1+\beta}}\right], \forall \xi \in E_{\mathbb{C}} .
$$

Then there exists a continuous operator $\Xi \in L\left((E)_{\beta},(E)_{\beta}\right)$ such that $G$ is the W-transform of $\Xi$.

Proof. The proof is similar to the proof of Theorem 4.1. So, we shall prove the existence of $\Xi$. Fix an arbitrary $\Phi \in(E)_{\beta}^{*}$. Define a $\mathbb{C}$-valued function $F_{\Phi}$ by

$$
F_{\Phi}(\xi)=\langle\langle\Phi, G(\xi)\rangle\rangle, \xi \in E_{\mathbb{C}} .
$$

Clearly, $F_{\Phi}$ satisfies conditions (1) and (2) in the Theorem 2.3. Hence, by Theorem 2.3, there exists a unique $\Psi_{\Phi} \in(E)_{\beta}^{*}$ such that

$$
S \Psi_{\Phi}(\xi)=\langle\langle\Phi, G(\xi)\rangle\rangle, \xi \in E_{\mathbb{C}} .
$$

Moreover, for any $r>p$ with

$$
e^{2}\left(\frac{2 a}{1+\beta}\right)^{1+\beta}\left\|A^{-(r-p)}\right\|_{H s}^{2}<1
$$




$$
\left\|\Psi_{\Phi}\right\|_{-r,-\beta} \leq K\|\Phi\|_{-q,-\beta}\left(1-\mathrm{e}^{2}\left(\frac{2 a}{1+\beta}\right)^{1+\beta}\left\|A^{-(r-p)}\right\|_{H s}^{2}\right)^{-1 / 2} .
$$

Therefore, the operator $\Phi \rightarrow \Psi_{\Phi}$ is continuous linear operator from $(E)_{\beta}^{*}$ into $(E)_{\beta}^{*}$. Now, let $\Xi$ be the adjoint of this operator, then $\Xi \in L\left((E)_{\beta},(E)_{\beta}\right)$ as desired.

\section{Convergence of Operator}

The convergence of operator sequences is rephrased in terms of convergence of $\mathrm{W}$-transform and symbol.

Theorem 4.1. Let $\{\Xi\}_{n=1}^{\infty}$ and $\Xi$ be in $L\left((E)_{\beta},(E)_{\beta}^{*}\right)$. Let $G_{n}=W \Xi_{n}, n \in \mathbb{N}$ and $G=W \Xi$. Then $\Xi_{n}$ converges to $\Xi$ strongly in $L\left((E)_{\beta},(E)_{\beta}^{*}\right)$ if and only if the following conditions are satisfied:

1) $G_{n}(\xi)$ converges to $G(\xi)$ in $(E)_{\beta}^{*}$ for each $\xi \in E_{\mathbb{C}}$.

2) There exist $q \geq 0, p \geq 0, K>0$ and $a>0$ such that

$$
\left\|G_{n}(\xi)\right\|_{-q,-\beta} \leq K \exp a\left(|\xi|_{p}^{\frac{2}{1-\beta}}\right), \xi \in E_{\mathbb{C}}, n \in \mathbb{N} .
$$

Proof. Suppose that $\Xi_{n}$ converges to $\Xi$ strongly in $L\left((E)_{\beta},(E)_{\beta}^{*}\right)$. Then for each $\phi \in(E)_{\beta}, \Xi_{n} \phi$ converges to $\Xi \phi$ in $(E)_{\beta}^{*}$. Clearly (1) is satisfied. To prove (2), we consider

$$
X_{q \beta, k}=\left\{\phi \in(E)_{\beta}, \sup _{n \in \mathbb{N}}\left\|\Xi_{n} \phi\right\|_{-q,-\beta} \leq k\right\} .
$$

Then we have $(E)_{\beta}=\bigcup_{q, \beta, k \in \mathbb{N}} X_{q, \beta, k}$. Since $(E)_{\beta}$ is a Frécht space, by the Baire's category theorem there exist $q$ and $k$ in $\mathbb{N}$ such that $X_{q, \beta, k}$ contains an open set of $(E)_{\beta}$. So we can see that there exist $p \in \mathbb{N}$ and $\varepsilon>o$ such that

$$
\left\{\phi \in(E)_{\beta} ;\|\phi\|_{p, \beta}<\varepsilon\right\} \subset X_{q, \beta, k} .
$$

Then for any $\phi \in(E)_{\beta}$, we have

$$
\left\|\Xi_{n} \phi\right\|_{-q,-\beta} \leq \frac{k}{\mathcal{E}^{\prime}}\|\phi\|_{p, \beta}
$$

for all $n \in \mathbb{N}$, where $0<\varepsilon^{\prime}<\varepsilon$. In particular, we have

$$
\left\|G_{n}(\xi)\right\|_{-q,-\beta}=\left\|\Xi_{n} \varphi_{\xi}\right\|_{-q,-\beta} \leq \frac{k}{\varepsilon^{\prime}}\left\|\varphi_{\xi}\right\|_{p, \beta} \leq \frac{k}{\varepsilon^{\prime}} \mathrm{e}^{a|\xi|_{p}^{\frac{2}{1-\beta}}} .
$$

This completes the proof of the first assertion.

Conversely, assume that $\left\{G_{n}\right\}$ satisfies the given conditions. Then by (1), for each $\xi \in E_{\mathbb{C}}$ and $\psi \in(E)_{\beta}$,

$$
\left\langle\left\langle\Xi_{n} \varphi_{\xi}, \psi\right\rangle\right\rangle \rightarrow\left\langle\left\langle\Xi \varphi_{\xi}, \psi\right\rangle\right\rangle \text {. }
$$

Since the linear span of $\left\{\varphi_{\xi} ; \xi \in E_{\mathbb{C}}\right\}$ is dense in $(E)_{\beta}$, it follows from (2) and Theorem 4.1 for any $\phi, \psi \in(E)_{\beta},\left\langle\left\langle\Xi_{n} \phi, \psi\right\rangle\right\rangle$ converges to $\langle\langle\Xi \phi, \psi\rangle\rangle$. This means that for any $\phi \in(E)_{\beta}, \Xi_{n} \phi$ converges to $\Xi \phi$ weakly in $(E)_{\beta}^{*}$. 
Hence, for any $\phi \in(E)_{\beta}, \Xi_{n} \phi$ converges to $\Xi \phi$ strongly in $(E)_{\beta}^{*}$. This completes the proof.

Corollary 4.2. let $\{\Xi\}_{n=1}^{\infty}$ and $\Xi$ be in $L\left((E)_{\beta},(E)_{\beta}^{*}\right)$. Let $F_{n}=\hat{\Xi}_{n}, n \in \mathbb{N}$ and $F=\hat{\Xi}$. Then $\Xi_{n}$ converges to $\Xi$ strongly in $L\left((E)_{\beta},(E)_{\beta}^{*}\right)$ if and only if the following conditions are satisfied:

1) For each $\xi, \eta \in E_{\mathbb{C}}, F_{n}(\xi, \eta)$ converges to $F(\xi, \eta)$.

2) There exist $p \geq 0, K>0$ and $a>0$ such that

$$
\left|F_{n}(\xi, \eta)\right| \leq K \exp a\left(|\xi|_{p}^{\frac{2}{1-\beta}}+|\eta|_{p}^{\frac{2}{1-\beta}}\right), \xi, \eta \in E_{\mathbb{C}}, n \in \mathbb{N} .
$$

Proof. To prove the Corollary, it suffices to prove that (1) and (2) in Theorem 4.1 are equivalent to (1) and (2). Now assume that (1) and (2) are satisfied. Using (2), we can see that for $\xi, \eta \in E_{\mathbb{N}}$ and for $n \in \mathbb{N}$,

$$
\left|S G_{n}(\xi)(\eta)\right|=\left|F_{n}(\xi, \eta)\right| \leq K \mathrm{e}^{\left.a|\xi|\right|_{p} ^{\frac{2}{1-\beta}}} \mathrm{e}^{a|\eta|_{p}^{\frac{2}{1-\beta}}} .
$$

Hence by Theorem 2.2, we have for any $q>p$ with

$$
\begin{gathered}
\mathrm{e}^{2}\left(\frac{2 a}{1-\beta}\right)^{1-\beta}\left\|A^{-(q-p)}\right\|_{H s}^{2}<1, \\
\left\|G_{n}(\xi)\right\|_{-q,-\beta} \leq K \mathrm{e}^{a \mid \xi \xi^{\frac{2}{1-\beta}}}\left(1-\mathrm{e}^{2}\left(\frac{2 a}{1-\beta}\right)^{1-\beta}\left\|A^{-(q-p)}\right\|_{H s}^{2}\right)^{-\frac{1}{2}}, \xi \in E_{\mathbb{C}}, n \in \mathbb{N} .
\end{gathered}
$$

On the other hand, using (1) we can show that for $\xi \in E_{\mathbb{N}}$, $\left\langle\left\langle G_{n}(\xi), \phi\right\rangle\right\rangle \rightarrow\langle\langle G(\xi), \phi\rangle\rangle$ for all $\phi \in(E)_{\beta}$. Hence conditions (1) and (2) are satisfied.

Theorem 4.3. let $\{\Xi\}_{n=1}^{\infty}$ and $\Xi$ be in $L\left((E)_{\beta},(E)_{\beta}\right)$. Let $G_{n}=W \Xi_{n}, n \in \mathbb{N}$ and $G=W \Xi$. Then $\Xi_{n}$ converges to $\Xi$ strongly in $L\left((E)_{\beta},(E)_{\beta}\right)$ if and only if the following conditions are satisfied:

1) For each $\xi \in E_{\mathbb{C}}, G_{n}(\xi)$ converges to $G(\xi)$ in $(E)_{\beta}$.

2) For each $q \geq 0$, there exist $p>0, K>0, a>0$ such that

$$
\left\|G_{n}(\xi)\right\|_{q, \beta} \leq K \exp a\left(|\xi|_{p}^{\frac{2}{1+\beta}}\right), \xi \in E_{\mathbb{C}}, n \in \mathbb{N} .
$$

Proof. Suppose that $\Xi_{n}$ converges to $\Xi \in L\left((E)_{\beta},(E)_{\beta}\right)$ strongly in $L\left((E)_{\beta},(E)_{\beta}\right)$. Then for any $\phi \in(E)_{\beta}, \Xi_{n} \phi$ converges to $\Xi \phi$ strongly in $(E)_{\beta}$. Hence (1) is obvious. To prove (2), given $q \geq 0$, we consider

$$
Y_{k}=\left\{\phi \in(E)_{\beta} ; \sup _{n \in \mathbb{N}}\left\|\Xi_{n} \phi\right\|_{q, \beta} \leq k\right\} .
$$

Then $Y_{k}$ is closed and $(E)_{\beta}=\bigcup_{k \in \mathbb{N}} Y_{k}$. Hence by using the similar arguments of the proof of Theorem 4.1, we can prove (2).

Conversely, assume that $\left\{G_{n}\right\}$ satisfies conditions (1) and (2). let $q \geq 0$, then by (1), we have

$$
\lim _{n \rightarrow \infty}\left\|\Xi_{n} \varphi_{\xi}-\Xi \varphi_{\xi}\right\|_{q, \beta}=0, \xi \in E_{\mathbb{C}}
$$


Hence, by using (2) and Theorem 4.4, we can prove that for any $\phi \in(E)_{\beta}$

$$
\lim _{n \rightarrow \infty}\left\|\Xi_{n} \phi-\Xi \phi\right\|_{q, \beta}=0 \text {. }
$$

This completes the proof.

Corollary 4.4. let $\{\Xi\}_{n=1}^{\infty}$ and $\Xi$ be in $L\left((E)_{\beta},(E)_{\beta}\right)$. Let $F_{n}=\hat{\Xi}_{n}, n \in \mathbb{N}$ and $F=\hat{\Xi}$. Then $\Xi_{n}$ converges to $\Xi$ strongly in $L\left((E)_{\beta},(E)_{\beta}\right)$ if and only if the following conditions are satisfied:

1) For each $\xi, \eta \in E_{\mathbb{C}}, F_{n}(\xi, \eta)$ converges to $F(\xi, \eta)$.

2) For each $q \geq 0$ and $a>0$, there exist $p \geq q, K>0$ such that

$$
\left|F_{n}(\xi, \eta)\right| \leq K \exp a\left(|\xi|_{p}^{\frac{2}{1+\beta}}+|\eta| \frac{2}{1+\beta}\right), \xi, \eta \in E_{\mathbb{C}}, n \in \mathbb{N} .
$$

Proof. The proof is straightforward by Corollary 4.2. We can prove that (1) and (2) in Theorem 4.3 are equivalent to (1) and (2).

\section{References}

[1] Chung, D.M., Chung, T.S. and Cig Ji., U. (1999) A Characterization Theorem for Operators on White Noise Functionals. Journal of the Mathematical Society of Japan, 51, 437-447. https://doi.org/10.2969/jmsj/05120437

[2] Hida, T. (1975) Analysis of Brownian Functionals. Carleton Mathematical Lecture Notes 13, Carleton University, Ottawa.

[3] Obata, N. (1993) An Analytic Characterization of Symbols of Operators on White Noise Functionals. Journal of the Mathematical Society of Japan, 45, 421-445. https://doi.org/10.2969/jmsj/04530421

[4] Kuo, H.H., Potthoff, J. and Streit, L. (1990) A Characterization of White Noise Test Functionals. Nagoya Mathematical Journal, 121, 185-194. https://doi.org/10.1017/S0027763000003469

[5] Potthoff, J. and Streit, L. (1991) A Characterization of Hida Distributions. Journal of Functional Analysis, 101, 212-229. https://doi.org/10.1016/0022-1236(91)90156-Y

[6] Obata, N. (1994) White Noise Calculus and Fock Space. Lecture Notes in Mathematics. Springer-Verlag, Berlin, 1577. https://doi.org/10.1007/BFb0073952

[7] Hida, T., Kuo, H.H., Potthoff, J. and Streit, L. (1995) White Noise: An Infinite Dimensional Calculus. Kluwer Academic Publishers, Dordrecht.

[8] Wang, C. and Chen, J. (2016) A Characterization of Operators on Functionals of Discrete-Time Normal Martingale. Stochastic Analysis and Applications, 35, 305-316. https://doi.org/10.1080/07362994.2016.1248779

[9] Kondratiev, Y.G. and Streit, L. (1993) Spaces of White Noise Distributions: Constructions, Descriptions, Applications I. Reports on Mathematical Physics, 33, 341-366. https://doi.org/10.1016/0034-4877(93)90003-W

[10] Kuo, H.H. (1996) White Noise Distribution Theory. CRC Press, Boca Raton. 